\title{
Keldysh-Rutherford Model for the Attoclock
}

\author{
Alexander W. Bray, ${ }^{1}$ Sebastian Eckart, ${ }^{2}$ and Anatoli S. Kheifets ${ }^{1}$ \\ ${ }^{1}$ Research School of Physics and Engineering, The Australian National University, \\ Canberra, Australian Capital Territory 0200, Australia \\ ${ }^{2}$ Institut für Kernphysik, Goethe-Universität, Max-von-Laue-Straße 1, 60438 Frankfurt, Germany
}

(Received 10 April 2018; published 20 September 2018)

\begin{abstract}
We demonstrate a clear similarity between attoclock offset angles and Rutherford scattering angles taking the Keldysh tunneling width as the impact parameter and the vector potential of the driving pulse as the asymptotic velocity. This simple model is tested against the solution of the time-dependent Schrödinger equation using hydrogenic and screened (Yukawa) potentials of equal binding energy. We observe a smooth transition from a hydrogenic to "hard-zero" intensity dependence of the offset angle with variation of the Yukawa screening parameter. Additionally, we make a comparison with the attoclock offset angles for various noble gases obtained with the classical-trajectory Monte Carlo method. In all cases we find a close correspondence between the model predictions and numerical calculations. This suggests a largely Coulombic origin of the attoclock offset angle and casts further doubt on its interpretation in terms of a finite tunneling time.
\end{abstract}

DOI: 10.1103/PhysRevLett.121.123201

Measuring an offset angle of the peak photoelectron momentum distribution in the polarization plane of a closeto-circularly polarized laser field has been used to determine the tunneling time which the photoelectron spends under the barrier [1-4]. Once the photoelectron leaves the tunnel at the peak value of the electric field, the most probable detection direction will be aligned with the vector potential at the instant of tunneling. This direction is tilted by $90^{\circ}$ relative to the electric field at this instant. A measurable angular offset from this axis can be converted to the time the photoelectron spends under the barrier with the conversion rate of 7.4 as $\left(1\right.$ as $\left.=10^{-18} \mathrm{~s}\right)$ per $1^{\circ}$ at $800 \mathrm{~nm}$. Such a measurement is termed colloquially the attoclock. A similar reading can be obtained from an attoclock driven by a very short circularly polarized laser pulse [5-9].

These attoclock measurements were made to address the controversy of a finite tunneling time, which has many decades of history [10]. This controversy is yet to be resolved with many conflicting reports of a finite time [11,12] as opposed to zero tunneling time $[5,6,9,13]$. A promising pathway to resolving this controversy is to examine an often neglected aspect of the attoclock measurement, the offset angle induced by the Coulomb field of the ion remainder. In theoretical calculations based on a numerical solution of the time-dependent Schrödinger equation (TDSE) [14-16], the Coulombic and tunneling components of the offset angle are inseparable. The Coulombic field's contribution can be switched off, however, by replacing the atomic potential with the short-range Yukawa potential of the same binding strength. This procedure effectively eliminates the offset angle, thus suggesting zero tunneling time $[5,13]$. In various classical (the TIPIS model [17], backpropagation [6,7],
CTMC [8]) or semiclassical simulations (the ARM model [5]), the Coulomb field is separable and its effect can be unambiguously determined.

If the laser field is not very strong and the pulse is sufficiently short, we can assess the Coulomb component of the offset angle by considering the motion of the photoelectron in the Coulomb field alone, decoupling it from the laser field altogether. Invoking scattering concepts in photoionization studies has been very fruitful over the years. The earliest and most relevant example is the Wigner group delay introduced initially for particle scattering [18] but more recently has found wide use in quantifying photoemission time delay in multiphoton ionization [19]. Further examples are multichannel scattering theories: the random phase approximation with exchange [20] and the convergent close-coupling theory [21]. The former describes electron scattering and photoionization of the closed-shell atoms while the latter solves the Coulomb three-body problem and describes single-photon double ionization of twoelectron atomic targets.

In the present work, we apply a similar concept: the photoelectron is tunnel ionized and then scatters elastically on the ion remainder. We examine cases where the applied field is relatively weak and the pulse is short, such that the actual trajectory of the field driven photoelectron is similar to that of an elastically scattered particle. This allows us to estimate the offset angle from the classical scattering formula [22]:

$$
\theta=2 \int_{r_{0}}^{\infty} \frac{\left(\rho / r^{2}\right) d r}{\left[1-(\rho / r)^{2}-\left(2 V / m v_{\infty}^{2}\right)\right]^{1 / 2}}-\pi
$$

Here, $\rho$ is the impact parameter, $v_{\infty}$ is the velocity of the projectile at the source and the detector, and the point of the 
closest approach $r_{0}$ is the largest positive root of the denominator. In the following we adopt the system of atomic units and set the charge and mass of the electron as well as the reduced Planck constant to unity $e=m=\hbar=1$. In the case of the attractive Coulomb potential $V(r)=-Z / r$, Eq. (1) takes the form of the Rutherford formula [22]:

$$
\tan \frac{\theta}{2}=\frac{1}{v_{\infty}^{2}} \frac{Z}{\rho} .
$$

In a more general case of a screened Coulomb potential $V(r)=-Z / r \exp (-r / \lambda)$, the offset angle is given by a modified expression [23] which is, up to a typically small numerical correction [see Eq. (13) of [23]],

$$
\tan \frac{\theta}{2}=\frac{1}{v_{\infty}^{2}} \frac{Z}{\rho} \exp \left(-1 / z_{0}\right)
$$

Here, $z_{0}$ is the root of $y(z)=1-(\rho / \lambda)^{2} z^{2}-(d / \lambda) z \exp (-1 / z)$ and $d=2 Z / v_{\infty}$ is the so-called collision diameter. If the last term in the right-hand side of $y(z)$ can be neglected, Eq. (3) acquires a simple screening exponent $\exp (-\rho / \lambda)$.

Equations (2) and (3) can be readily applied to the case of tunneling ionization. First, we note that photoionization can be considered as half-scattering and the offset angle should be taken as one half of the Rutherford or Yukawa scattering angles. Similarly, the Wigner time delay in photoemission is equated with the energy derivative of the scattering phase, whereas it is twice this value in electron scattering. Second, the point of the closest approach $r_{0}$ should be taken as the tunnel exit position which, for the Coulomb potential, is the largest root of the equation

$$
Z / r+E_{0} r=I_{p}, \quad r_{1,2}=b / 2 \pm \sqrt{b^{2} / 4-a b} .
$$

Here, $I_{p}$ is the ionization potential, $E_{0}$ is the peak value of the electric field, $b=I_{p} / E_{0}$, and $a=Z / I_{p}$. In the weak field limit, $r_{1}=b \gg r_{2}=a \simeq 1$, where $a$ is the characteristic span of the atomic orbital. The onset of the over-the-barrier ionization corresponds to $r_{1}=r_{2}$ and $E_{0}=I_{p}^{2} /(4 Z)$ (righthand panel of Fig. 1). We note that the $b$ parameter is used to evaluate the Keldysh tunneling time $\tau=b / v_{\text {at }}$ with $v_{\text {at }}=$ $\sqrt{2 I_{p}}$ which, in turn, defines the adiabaticity parameter $\gamma=\omega \tau$ [24]. Finally, we equate the asymptotic velocity with the value of the vector potential at the moment of ionization, $v_{\infty}=A_{0}$. In the case of adiabatic tunneling, the longitudinal velocity of the tunneled electron in the direction of the electric field $\boldsymbol{E}_{0}$ at the point of exit is zero. Similarly, the radial velocity of the scattering electron at the point of the closest approach $r_{0}$ is also vanishing.

To proceed further, we assume that $\rho \simeq r_{0}$, which is a reasonable approximation in the case of small scattering angles. This also entails $\tan (\theta / 2) \simeq \theta / 2$. In addition to this we neglect the influence of the central potential at the
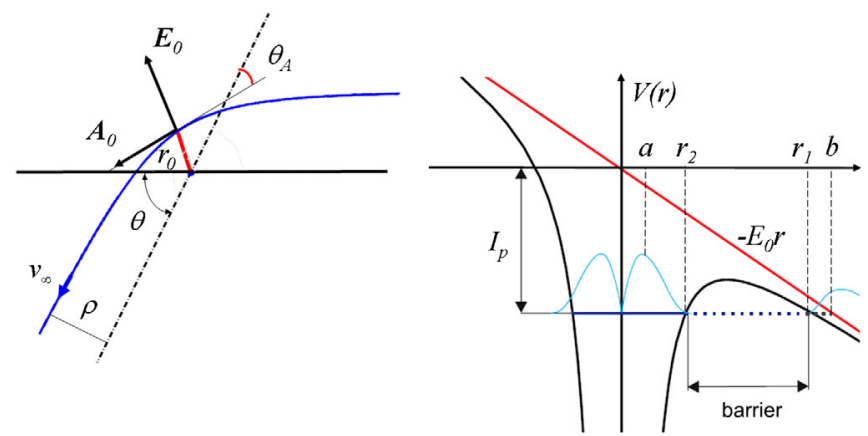

FIG. 1. Left: Classical scattering trajectory of a particle in a central attractive potential. The scattering angle $\theta$ is defined by the impact parameter $\rho$ and the asymptotic velocity $v_{\infty}$. The tunnel ionized electron enters this trajectory at the point of the closest approach $r_{0}$ driven by the peak electric field $\boldsymbol{E}_{0}$ and arriving to the detector at the angle $\theta_{A}$ relative to the vector potential $\boldsymbol{A}_{0}$. Right: The Coulomb potential is tipped by the light field. A finite width potential barrier is created, through which the electron wave packet leaks out. $I_{p}$ refers to the binding energy of the electron in an unperturbed atomic system. See text for further symbol definitions.

tunnel exit and simply set $r_{0}=b$. With these assumptions, the attoclock offset angle in the case of the pure Coulomb potential takes the form

$$
\theta_{A}=\frac{1}{2} \theta \simeq \frac{\omega^{2}}{E_{0}^{2}} \frac{Z}{\rho}=\frac{\omega^{2}}{E_{0}} \frac{Z}{I_{p}} .
$$

This equation allows for an alternative interpretation. Indeed, the Rutherford formula Eq. (2) can be rewritten as

$$
\tan \frac{\theta}{2}=\frac{1}{v_{\infty} \rho} \frac{Z}{v_{\infty}}=\frac{1}{L} \frac{Z}{v_{\infty}}=\frac{Z}{L} \frac{\omega}{E_{0}},
$$

where $L$ is the angular momentum of the projectile. In the attoclock experiment with circular polarized light, each photon absorption adds one unit of angular momenta and, hence, $L \simeq I_{p} / \omega$. This immediately leads to Eq. (5).

The signature of this Keldysh-Rutherford (KR) formula is the field intensity dependence of the offset angle $\theta_{A} \propto E_{0}^{-1} \propto I^{-1 / 2}$. This dependence can be understood as a result of competition of the two terms in Eq. (2): the kinetic energy term $v_{\infty}^{2} / 2$ and the potential energy term $Z / \rho$. As the field intensity grows, the kinetic energy grows linearly with $I$. Hence, this term alone would result in a $I^{-1}$ dependence of the offset angle. This is partially compensated by the potential energy term as the width of the barrier decreases as $I^{-1 / 2}$. Hence, the resulting offset angle also decreases as $I^{-1 / 2}$. The signature of the over-the-barrier ionization regime is a $I^{-1}$ dependence as the tunnel width no longer depends on the field intensity.

The recent attoclock measurement on the hydrogen atom [13] confirmed the $I^{-1 / 2}$ dependence experimentally. 
This measurement, however, used relatively long pulses without carrier envelope phase stabilization. In addition, the offset angle extraction procedure was rather complicated due to experimental constraints. As such, this data set does not provide a good reference to test the KR model against. Instead, we conduct our own "numerical experiment" in a similar manner to that of Ref. [5]. We solve numerically the TDSE,

$$
i \partial \Psi(\boldsymbol{r}) / \partial t=\left[\hat{H}_{\text {atom }}+\hat{H}_{\text {int }}(t)\right] \Psi(\boldsymbol{r}),
$$

where $\hat{H}_{\text {atom }}$ describes the atomic target in the absence of the applied field, and the interaction Hamiltonian is written in the velocity gauge:

$$
\hat{H}_{\mathrm{int}}(t)=\boldsymbol{A}(t) \cdot \hat{\boldsymbol{p}}, \quad \boldsymbol{E}(t)=-\partial \boldsymbol{A} / \partial t .
$$

The vector potential of the driving pulse we take as

$$
\boldsymbol{A}(t)=-A_{0} f(t)\left[\cos (\omega t) \hat{e}_{x}+\sin (\omega t) \hat{e}_{y}\right]
$$

with the envelope function $f(t)=\cos ^{4}(\omega t / 4)$ for $-2 \pi / \omega<t<2 \pi / \omega$ and zero elsewhere. The (peak) field intensity is given by $I=2\left(\omega A_{0}\right)^{2}$ and the frequency $\omega \simeq$ 0.057 a.u. corresponds to $800 \mathrm{~nm}$ radiation. At the tunneling instant $t=0$ the electric field $E_{0}$ reaches its maximum in the $\hat{e}_{y}$ direction, whereas the vector potential $A_{0}$ is largest in the $-\hat{e}_{x}$ direction. The rotating electric field of the driving pulse causes the photoelectron to make a single turn by $90^{\circ}$ before it arrives to the detector in the $\hat{e}_{x}$ direction with momentum $A_{0}$. Photoelectron scattering in the Coulomb field adds an offset angle $\theta_{A}$ relative to this direction (left-hand panel of Fig. 1).

Solution of the TDSE [Eq. (7)] is found using the infinite-time surface flux (iSURF) method implemented in Ref. [25]. A typical calculation takes around $140 \mathrm{CPU}$ hours on a high-performance, distributed-memory cluster. The solution of the TDSE is projected on the scattering states of the target atom, thus forming the photoelectron momentum distribution. A typical 2D momentum distribution in the polarization plane $k^{2} P\left(k_{x}, k_{y}\right)$ is shown in Fig. 2. This distribution is integrated radially to obtain the angular distribution $P(\theta)=\int d k k^{2} P\left(k_{x}, k_{y}\right)$. It is then fitted with a Gaussian to determine the peak position and this value assigned to the attoclock offset angle $\theta_{A}$. The symmetry of $P(\theta)$ relative to $\theta_{A}$ is carefully monitored and serves as a test of the quality of the TDSE calculation.

Resulting values of the offset angle $\theta_{A}$ for hydrogen at various field intensities are plotted in Fig. 3. We find the present set of TDSE calculations to be hardly distinguishable from the set labeled H2 of TDSE calculations reported in Ref. [5]. This is contrasted with the two KR and $\mathrm{KR}^{\prime}$ estimates. For the former we simply plot Eq. (5), whereas in the latter we do not make the small angle approximation for

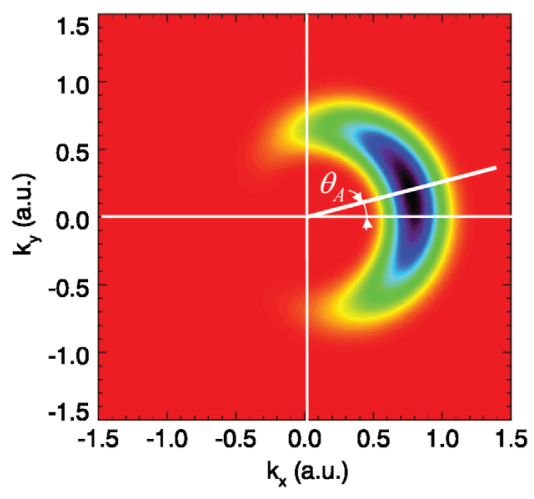

FIG. 2. Photoelectron momentum distribution in the polarization plane at a driving field intensity of $I=8.6 \times 10^{13} \mathrm{~W} / \mathrm{cm}^{2}$ on hydrogen. The offset angle $\theta$ relative to the vector potential direction at the instant of tunnel is marked. The coloration ranges from zero (red) to the maximum amplitude (black) linearly.

the tangent function. Accordingly, both estimates converge together with increasing field intensity. The KR scales at all intensities as $I^{-0.5}$ by construction. Fitting the $\mathrm{KR}^{\prime}$ in the low intesity range yields $I^{-0.44}$. The TDSE results display a similar dependency of $I^{-0.41}$ scaling for the same region but then flatten and deviate from both the KR and $\mathrm{KR}^{\prime}$. This is understandable as the KR model is expected to be valid only for weak fields where the field driven trajectory is close to that involved in field-free scattering.

In Fig. 4 we present the offset angles for a model Yukawa atom as a function of the driving pulse intensity. The top panel contains our TDSE results, whereas the bottom panel displays the predictions of the $\mathrm{KY}^{\prime}$ model, identical to that of $\mathrm{KR}^{\prime}$ but instead based on Eq. (3). Each curve on these panels correspond to a given value of the screening constant $\lambda$, which varies from a purely Coulombic case $\lambda=\infty$, to the severe screening $\lambda=1$ considered in Refs. [5,13]. In all cases, the nuclear charge $Z$ is chosen such that the

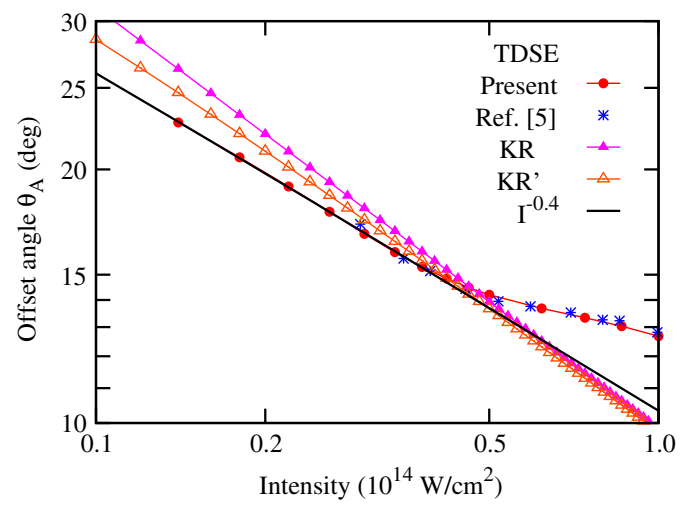

FIG. 3. The attoclock offset angle $\theta_{A}$ as a function of the field intensity $I$ from the present set of TDSE calculations on hydrogen (red filled circles), the set labeled H2 of Ref. [5] (blue asterisks), and the $\mathrm{KR}$ and $\mathrm{KR}^{\prime}$ models (filled and empty triangles). The present TDSE results are fitted with $I^{-0.41}$. 


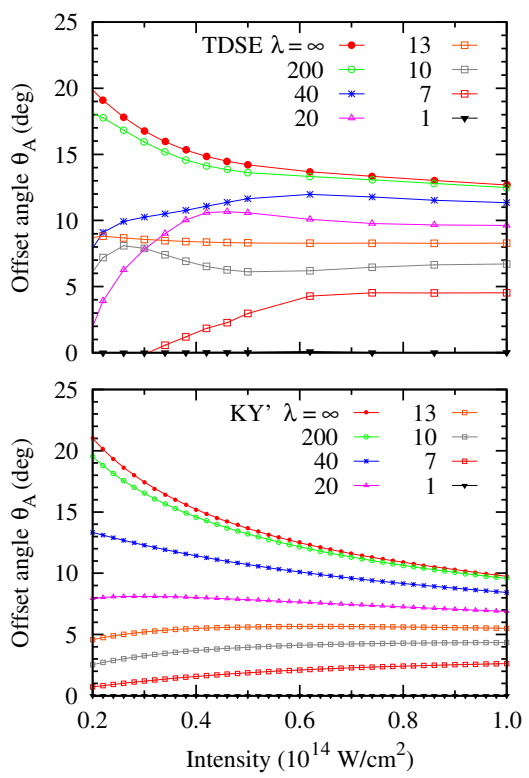

FIG. 4. The attoclock offset angle as a function of the field intensity for a model Yukawa atom with different screening constants $\lambda$. Top: TDSE calculations. Bottom: Predictions of the $\mathrm{KY}^{\prime}$ model.

ionization potential remains equal to that of the hydrogen atom $I_{p}=0.5$. At smaller $\lambda$, the TDSE results shown on the top panel of Fig. 4 do not follow predictions of the KY' model faithfully. This occurs as with decreasing intensity the photoelectron momentum, and associated de Broglie wavelength, becomes comparable to the screening length and hence the scattering process cannot be considered classical [23]. Nevertheless, both sets of curves are qualitatively very similar. We observe that the field intensity dependence of the offset angle for a Yukawa atom is drastically different from the purely Coulombic case. Instead of the growth with decreasing field strength, the offset angle becomes smaller. This is understandable as the tunnel width $b=I_{p} / E_{0}$ grows and becomes comparable with the screening length $\lambda$. In this regime, the offset angle is exponentially cut off. For the field intensity of $I=5 \times 10^{13} \mathrm{~W} / \mathrm{cm}^{2}, b \simeq 20$. Accordingly, we observe a significant decrease of the offset angle at and below this field intensity for the Yukawa atom with screening length $\lambda=20$. Finally, the offset angles are essentially zero in both the TDSE and $\mathrm{KY}^{\prime}$ in the case of severe screening $\lambda=1$, as in Refs. [5,13].

Although the KR model is developed explicitly for hydrogenic targets, it can be easily applied to other atoms. Indeed, the asymptotic charge affecting the departing photoelectron is always the same for all neutral atomic systems. Hence, the basic premise of the KR model remains valid. To test this model for other atoms, we choose an extended set of attoclock simulations [8] conducted using the classical-trajectory Monte Carlo (CTMC) method. We fit the CTMC offset angles with the KR ansatz,

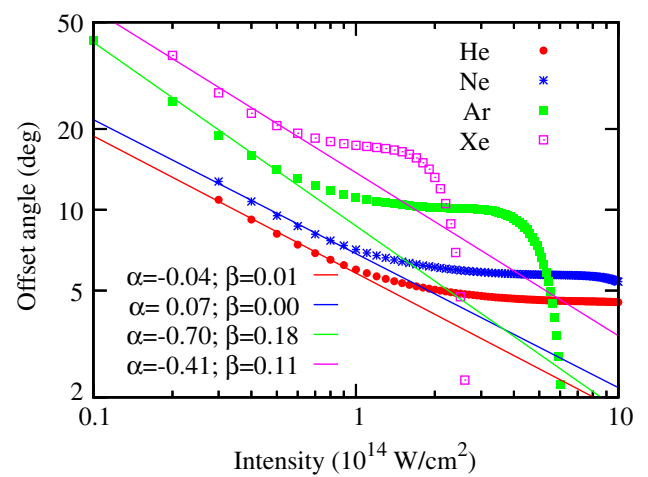

FIG. 5. The attoclock offset angles from CTMC calculations [8] as functions of the field intensity (data points) are fitted with the KR ansatz Eq. (10) (similarly colored solid lines).

$$
\theta_{A}(I)=\frac{\omega^{2}}{I_{p}} \frac{(1+\alpha)}{\left(I / 2 I_{0}\right)^{0.5+\beta}}
$$

where $I_{0}=3.51 \times 10^{16} \mathrm{~W} / \mathrm{cm}^{2}$ is 1 a.u. of field intensity. We use $\alpha$ and $\beta$ as fitting parameters which indicate the deviation of the CTMC calculation from the KR predictions. Results of the ansatz Eq. (10) application are illustrated in Fig. 5. We see that for small intensities the scaling of the offset angles with the field intensity is indeed close to $I^{-0.5}$. There is most deviation from the KR prediction in $\mathrm{Ar}$ and $\mathrm{Xe}$, where the fitting parameters are comparatively large. These parameters are in contrast near zero for the targets with larger ionization potentials, $\mathrm{He}$ and $\mathrm{Ne}$.

In conclusion, we derived a simple empirical expression for the attoclock offset angle based on the classical Rutherford scattering formula and the Keldysh estimate of the tunnel width. Utility of these formulas is demonstrated through application to hydrogen and Yukawa atoms in addition to noble gas atoms driven by short circularly polarized pulses. Because of its simplicity, the KeldyshRutherford formula can be easily applied to attoclock experiments with arbitrary polarization though modification of the above formalism to account for nonunitary ellipticity. One such case being the recent attoclock measurements on atomic hydrogen [13], where the signature field intensity scaling of the KR model $I^{-0.5}$ was indeed observed. These measurements as well as the earlier "numerical experiment" [5] were indicative of a zero tunneling time. This interpretation followed from the TDSE simulations with the screened Yukawa potential with $\lambda=1$, which set the attoclock offset angles to zero independently of the field intensity. In the present work we show that this interpretation is overly simplistic. Indeed, the crossover from the hydrogen to Yukawa atoms is smooth, and by decreasing the screening length $\lambda$ one can switch off the action of the Coulomb field gradually. In fact, it is only the shortest screening length $\lambda=1$ considered here that sends the offset angles below $1^{\circ}$. Nonetheless, in each case we find the offset angles to follow closely the predictions of the KR model and as such are not, 
most likely, related to the time the tunneled electron spends under the barrier.

The authors are very thankful to Satya Sainadh, Nicolas Duguet, Igor Ivanov, Klaus Bartschat, Igor Litvinyuk, and Robert Sang for many stimulating discussions. The authors are also greatly indebted to Serguei Patchkovskii who placed his iSURF TDSE code at their disposal. Finally, we thank Zengxiu Zhao for supplying the data [8] in numerical form. S. E. was supported by the DFG Priority Programme DO 604/29-1 and A. S. K. by the Wilhelm and Else Heraeus Foundation. Resources of the National Computational Infrastructure were employed.

[1] P. Eckle, M. Smolarski, P. Schlup, J. Biegert, A. Staudte, M. Schöffler, H. G. Muller, R. Dörner, and U. Keller, Attosecond angular streaking, Nat. Phys. 4, 565 (2008).

[2] P. Eckle, A. N. Pfeiffer, C. Cirelli, A. Staudte, R. Dorner, H. G. Muller, M. Buttiker, and U. Keller, Attosecond ionization and tunneling delay time measurements in helium, Science 322, 1525 (2008).

[3] A. N. Pfeiffer, C. Cirelli, M. Smolarski, D. Dimitrovski, M. Abu-samha, L. B. Madsen, and U. Keller, Attoclock reveals natural coordinates of the laser-induced tunnelling current flow in atoms, Nat. Phys. 8, 76 (2012).

[4] A. S. Landsman, M. Weger, J. Maurer, R. Boge, A. Ludwig, S. Heuser, C. Cirelli, L. Gallmann, and U. Keller, Ultrafast resolution of tunneling delay time, Optica 1, 343 (2014).

[5] L. Torlina, F. Morales, J. Kaushal, I. Ivanov, A. Kheifets, A. Zielinski, A. Scrinzi, H. G. Muller, S. Sukiasyan, M. Ivanov et al., Interpreting attoclock measurements of tunnelling times, Nat. Phys. 11, 503 (2015).

[6] H. Ni, U. Saalmann, and J.-M. Rost, Tunneling Ionization Time Resolved by Backpropagation, Phys. Rev. Lett. 117, 023002 (2016).

[7] H. Ni, U. Saalmann, and J.-M. Rost, Tunneling exit characteristics from classical backpropagation of an ionized electron wave packet, Phys. Rev. A 97, 013426 (2018).

[8] J. Liu, Y. Fu, W. Chen, Z. Lu, J. Zhao, J. Yuan, and Z. Zhao, Offset angles of photocurrents generated in few-cycle circularly polarized laser fields, J. Phys. B 50, 055602 (2017).

[9] N. Eicke and M. Lein, Trajectory-free ionization times in strong-field ionization, Phys. Rev. A 97, 031402 (2018).

[10] R. Landauer and T. Martin, Barrier interaction time in tunneling, Rev. Mod. Phys. 66, 217 (1994).
[11] A. S. Landsman and U. Keller, Attosecond science and the tunnelling time problem, Phys. Rep. 547, 1 (2015).

[12] N. Camus, E. Yakaboylu, L. Fechner, M. Klaiber, M. Laux, Y. Mi, K. Z. Hatsagortsyan, T. Pfeifer, C. H. Keitel, and R. Moshammer, Experimental Evidence for Quantum Tunneling Time, Phys. Rev. Lett. 119, 023201 (2017).

[13] U.S. Sainadh, H. Xu, X. Wang, Atia-Tul-Noor, W. C. Wallace, N. Douguet, A. W. Bray, I. Ivanov, K. Bartschat, A. Kheifets et al., Attosecond angular streaking and tunnelling time in atomic hydrogen, arXiv:1707.05445v1.

[14] C. P. J. Martiny, M. Abu-samha, and L. B. Madsen, Counterintuitive angular shifts in the photoelectron momentum distribution for atoms in strong few-cycle circularly polarized laser pulses, J. Phys. B 42, 161001 (2009).

[15] N. I. Shvetsov-Shilovski, D. Dimitrovski, and L. B. Madsen, Ionization in elliptically polarized pulses: Multielectron polarization effects and asymmetry of photoelectron momentum distributions, Phys. Rev. A 85, 023428 (2012).

[16] I. A. Ivanov and A. S. Kheifets, Strong-field ionization of He by elliptically polarized light in attoclock configuration, Phys. Rev. A 89, 021402 (2014).

[17] R. Boge, C. Cirelli, A. S. Landsman, S. Heuser, A. Ludwig, J. Maurer, M. Weger, L. Gallmann, and U. Keller, Probing Nonadiabatic Effects in Strong-Field Tunnel Ionization, Phys. Rev. Lett. 111, 103003 (2013).

[18] E. P. Wigner, Lower limit for the energy derivative of the scattering phase shift, Phys. Rev. 98, 145 (1955).

[19] R. Pazourek, S. Nagele, and J. Burgdörfer, Attosecond chronoscopy of photoemission, Rev. Mod. Phys. 87, 765 (2015).

[20] M. Y. Amusia, Atomic Photoeffect (Plenum Press, New York, 1990).

[21] I. Bray, D. Fursa, A. Kadyrov, A. Stelbovics, A. Kheifets, and A. Mukhamedzhanov, Electron- and photon-impact atomic ionisation, Phys. Rep. 520, 135 (2012).

[22] L. Landau and E. Lifshitz, Mechanics, in Theorecial Physics Vol. 1 (Elsevier, Butterworth-Heineman, Oxford, UK, 1982).

[23] E. Everhart, G. Stone, and R. J. Carbone, Classical calculation of differential cross section for scattering from a Coulomb potential with exponential screening, Phys. Rev. 99, 1287 (1955).

[24] S. V. Popruzhenko, Keldysh theory of strong field ionization: History, applications, difficulties and perspectives, J. Phys. B 47, 204001 (2014).

[25] F. Morales, T. Bredtmann, and S. Patchkovskii, iSURF: A family of infinite-time surface flux methods, J. Phys. B 49, 245001 (2016). 